Gut and Liver, Vol. 10, No. 5, September 2016, pp. 731-738

\title{
Prognosis of Pregnancy-Associated Gastric Cancer: An Age-, Sex-, and Stage-Matched Case-Control Study
}

Min Jeong Song ${ }^{1}$, Young Soo Park ${ }^{1}$, Ho June Song ${ }^{2}$, Se Jeong Park², Ji Yong Ahn ${ }^{2}$, Kee Don Choi ${ }^{2}$, Gin Hyug Lee², HwoonYong Jung ${ }^{2}$, Jeong Hwan Yook ${ }^{3}$, and Byung Sik Kim ${ }^{3}$

Departments of ${ }^{1}$ Pathology, ${ }^{2}$ Internal Medicine, and ${ }^{3}$ Surgery, Asan Medical Center, University of Ulsan College of Medicine, Seoul, Korea

Background/Aims: Pregnancy-associated gastric cancer is a rare condition. This case-control study was performed to identify the clinicopathological features and prognostic factors of pregnancy-associated gastric cancer. Methods: All consecutive patients who presented to our tertiary referral hospital with pregnancy-associated gastric cancer from 1991 to 2012 were identified. Two age-, sex-, and stagematched controls for each case were also identified from the records. Clinicopathological, gynecological, and oncological outcomes were recorded. Immunohistochemical staining was performed for estrogen receptor, progesterone receptor, epidermal growth factor receptor, human epidermal growth factor receptor, and E-cadherin. Fluorescence in situ hybridization was performed for fibroblast growth factor receptor 2 . Results: The median overall survival rates of the pregnancyassociated gastric cancer and control groups were 7.0 months and 15.0 months, respectively $(p=0.189)$. Poor prognostic factors included advanced stage and tumor location in the corpus or the entire stomach but not pregnancy status or loss of E-cadherin. Pregnancy-associated gastric cancer was associated with a longer time from diagnosis to treatment (21 days vs 7 days, $p=0.021$ ). The two groups did not differ in the expression of the receptors or E-cadherin. Conclusions: The dismal prognosis of pregnancy-associated gastric cancer may related to the tumor stage and location rather than to pregnancy itself. (Gut Liver 2016;10:731-738)

Key Words: Gastric neoplasms; Pregnancy; Prognosis; Ecadherins

\section{INTRODUCTION}

Although the incidence of gastric cancer is decreasing worldwide, ${ }^{1}$ it remains a leading cause of cancer-related mortality, particularly in Asian countries. ${ }^{2}$ While most patients are middleaged or elderly, approximately 10\% are under 45 years of age. ${ }^{3}$ Pregnancy-associated gastric cancer, which is diagnosed during pregnancy or the lactation period, is even rarer, accounting for only $0.025 \%$ to $0.1 \%$ of all pregnancies. ${ }^{4}$ Despite its rarity, gastric cancer diagnosed during pregnancy can be a devastating clinical situation for both the mother and the fetus: patients with gastric cancer diagnosed during pregnancy have a dismal prognosis. ${ }^{4-7}$ This may be partly due to the fact that most pregnancy-associated gastric cancers are diagnosed at an advanced stage and that only $45 \%$ to $56 \%$ of the patients undergo surgical resection. ${ }^{4,5}$ A possible explanation for this is that patients under 40 years are not included in the gastric cancer program in Korea, ${ }^{8}$ and symptoms such as nausea and/or vomiting or abdominal discomfort during pregnancy are generally overlooked or not considered serious.

Pregnancy-associated gastric cancer is characterized by a high incidence of Borrmann type IV gross type, frequent peritoneal seeding, diffuse-type histology (according to Lauren classification), and frequent signet ring cell features. ${ }^{6}$ Interestingly, these clinicopathological features overlap with those of gastric cancers in younger patients, which occur more frequently in females. ${ }^{9-11}$ The female predominance in young-age gastric cancers along with the fact that females with such cancers have a lower survival rate than males suggest that hormonal factors such as estrogen may promote the development of more dangerous gastric cancers. ${ }^{11}$ It has also been reported that pregnan-

Correspondence to: Young Soo Park ${ }^{\mathrm{a}}$ and Ho June Song ${ }^{\mathrm{b}}$

${ }^{a}$ Department of Pathology, Asan Medical Center, University of Ulsan College of Medicine, 88 Olympic-ro 43-gil, Songpa-gu, Seoul 05505, Korea Tel: +82-2-3010-5608, Fax: +82-2-472-7898, E-mail: youngspark@amc.seoul.kr

${ }^{b}$ Department of Internal Medicine, Asan Medical Center, University of Ulsan College of Medicine, 88 Olympic-ro 43-gil, Songpa-gu, Seoul 05505, Korea

Tel: +82-2-3010-3916, Fax: +82-2-2045-4043, E-mail: hjsong@amc.seoul.kr

Received on July 13, 2015. Revised on October 1, 2015. Accepted on November 20, 2015. Published online April 28, 2016

pISSN 1976-2283 eISSN 2005-1212 http://dx.doi.org/10.5009/gnl15323

() This is an Open Access article distributed under the terms of the Creative Commons Attribution Non-Commercial License (http://creativecommons.org/licenses/by-nc/4.0) which permits unrestricted non-commercial use, distribution, and reproduction in any medium, provided the original work is properly cited. 
cy-associated gastric cancers have a worse prognosis because of a delay in diagnosis and a passive attitude toward treatment. ${ }^{4,5,7}$ Therefore, it is not clear whether the pregnancy itself exerts a negative influence on the prognosis of gastric cancer.

In the present case-control study, patients with pregnancyassociated gastric cancers and age-, sex-, and stage-matched control cases were compared to identify prognostic clinicopathological factors. In addition, the expression of E-cadherin and several growth factor receptors, namely, estrogen receptor (ER), progesterone receptor (PR), epidermal growth factor receptor (EGFR), human epidermal growth factor receptor 2 (Her2), and fibroblast growth factor receptor 2 (FGFR2), were assessed.

\section{MATERIALS AND METHODS}

\section{Patients and data collection}

All consecutive patients with pregnancy-associated malignancy who presented to our tertiary referral hospital in Seoul, Korea (Asan Medical Center) between January 1991 and December 2012 were identified by retrospective database review. Pregnancy-associated malignancy was defined as any malignancy that was diagnosed during pregnancy or the lactation period (i.e., within 2 months after the birth). ${ }^{5}$ The patients who had gastric carcinoma were then identified. These patients formed the case cohort of the study. Relevant clinical information, including endoscopic findings, gynecological status, obstetric management, treatment, and outcome of gastric carcinoma, were recorded. In addition, for each case, two age- $( \pm 2$ years), sex-, and TNM stage-matched control patients who presented to Asan Medical Center in 1991 to 2012 were sought. However, only one control could be found for case 2. Thus, in total, there were 39 controls (Table 1). All tumor tissue specimens, including biopsies, cytology, and surgical specimens, were reviewed by two pathologists (M.J.S. and Y.S.P.).

\section{Immunohistochemistry and fluorescence in situ hybrid- ization}

Immunohistochemistry (IHC) for ER, PR, EGFR, Her2, and Ecadherin were performed using a BenchMark XT automated immunostaining system (Ventana Medical System, Tucson, AZ, USA). Briefly, $4 \mu \mathrm{m}$-thick sections were transferred onto adhesive slides and dried at $62^{\circ} \mathrm{C}$ for 30 minutes. Following standard heat epitope retrieval for 60 minutes in ethylenediaminetetraacetic acid, $\mathrm{pH}$ 8.0, in the autostainer, the samples

Table 1. Clinical Features and Outcomes of Gastric Cancers Associated with Pregnancy

\begin{tabular}{|c|c|c|c|c|c|c|c|c|}
\hline \multirow{2}{*}{$\begin{array}{l}\text { Case } \\
\text { no. }\end{array}$} & \multirow{2}{*}{$\begin{array}{l}\text { Age, } \\
\text { yr }\end{array}$} & \multirow{2}{*}{$\begin{array}{c}\text { GA at } \\
\text { diagnosis }\end{array}$} & \multirow[b]{2}{*}{ Presentation } & \multirow[b]{2}{*}{ Obstetric outcome (GA) } & \multirow[b]{2}{*}{ GC stage* } & \multirow[b]{2}{*}{ GC treatment } & \multicolumn{2}{|c|}{ Survival outcome } \\
\hline & & & & & & & $\begin{array}{l}\text { Maternal (time } \\
\text { after diagnosis) }\end{array}$ & Infant \\
\hline 1 & 23 & $9 \mathrm{wk}$ & Nausea/vomiting & Termination (13 wk) & II (pT4NOM0) & Gastrectomy+CTx & Death (3.4 yr) & Death \\
\hline 2 & 26 & +28 day & Abdominal pain & Delivery (42 wk) & IV (pT4NxM1) & Supportive care & Death (2 mo) & Alive \\
\hline 3 & 26 & $29 \mathrm{wk}$ & Ascites & Delivery (40 wk) & IV (cTxNxM1) & Supportive care & Death (2 mo) & Alive \\
\hline 4 & 27 & $28 \mathrm{wk}$ & Neck mass & Termination (30 wk) & IV (pTxNxM1) & Palliative CTx & Death (2 wk) & Death \\
\hline 5 & 29 & $34 \mathrm{wk}$ & Jaundice & Delivery (38 wk) & IV (cTxNxM1) & Supportive care & Death (4 mo) & Alive \\
\hline 6 & 29 & $35 \mathrm{wk}$ & Ascites & Cesarean section (35 wk) & IV (pTxNxM1) & Palliative CTx & Death (8 mo) & Alive \\
\hline 7 & 30 & $28 \mathrm{wk}$ & Melena & Cesarean section (30 wk) & II (pT4NOMO) & Gastrectomy & Alive (15 mo) & Alive \\
\hline 8 & 31 & 33 wk & Chest pain & Delivery (34 wk) & IV (pTxNxM1) & Palliative CTx & Death (3 mo) & Alive \\
\hline 9 & 31 & +3 day & Ascites & Delivery (35 wk) & IV (cTxNxM1) & Palliative CTx & Death (3 mo) & Alive \\
\hline 10 & 31 & +10 day & Nausea/vomiting & Cesarean section (31 wk) & IV (cTxNxM1) & Supportive care & Death (23 mo) & Alive \\
\hline 11 & 31 & $32 \mathrm{wk}$ & Nausea/vomiting & Cesarean section (32 wk) & IV (cTxNxM1) & Supportive care & Death (6 wk) & Alive \\
\hline 12 & 31 & $38 \mathrm{wk}$ & Flank pain & Cesarean section (38 wk) & IV (cTxNxM1) & Palliative CTx & Death (15 mo) & Alive \\
\hline 13 & 32 & 26 wk & Abdominal pain & Cesarean section (27 wk) & IV (cTxNxM1) & Palliative CTx & Death (6 mo) & Alive \\
\hline 14 & 32 & $26 \mathrm{wk}$ & Dyspepsia & Cesarean section (28 wk) & I (pT1N1M0) & Gastrectomy & Alive (26 mo) & Alive \\
\hline 15 & 33 & $38 \mathrm{wk}$ & Nausea/vomiting & Delivery (38 wk) & IV (pTxNXM1) & Palliative CTx & Death (7 mo) & Alive \\
\hline 16 & 34 & $1 \mathrm{wk}$ & Dyspepsia & Termination (7 wk) & III (pT4N3M0) & Gastrectomy+CTx & Death (24 mo) & Death \\
\hline 17 & 35 & $8 \mathrm{wk}$ & Abdominal pain & Termination (8 wk) & IV (cTxNxM1) & Palliative CTx & Death (6 mo) & Death \\
\hline 18 & 37 & $5 \mathrm{wk}$ & Dyspepsia & Termination (5 wk) & II (pT1N2M0) & Gastrectomy+CTx & Alive (17 yr) & Death \\
\hline $19^{\dagger}$ & 38 & $21 \mathrm{wk}$ & Abdominal pain & Termination (23 wk) & IV (pTxNxM1) & Supportive care & Death (1 mo) & Death \\
\hline 20 & 39 & $5 \mathrm{wk}$ & Abdominal pain & Termination (7 wk) & III (pT4N3M0) & Gastrectomy+CTx & Alive (33 mo) & Death \\
\hline
\end{tabular}

GA, gestational age; GC, gastric cancer; +, diagnosed during the postpartum period; CTx, chemotherapy.

${ }^{*}$ Clinical stages according to the American Joint Committee on Cancer, seventh edition; ${ }^{\dagger}$ This case exhibited placental metastasis. 
were incubated with antibodies to ER (clone 6F11; Novocastra Laboratories, Newcastle upon Tyne, UK), PR (clone16; Novocastra Laboratories), EGFR (clone 31G7; Zymed Laboratories, South San Francisco, CA, USA), Her2 (clone 4B5; Ventana Medical Systems), or E-cadherin (clone4 A2C7; Invitrogen, Paisley, UK). After incubation with primary antibodies, the sections were subsequently incubated with an ultraView universal DAB kit (Ventana Medical Systems). Appropriate positive controls were mounted on each slide for each antibody. Slides were counterstained with Harris hematoxylin. The ER and PR slides were scored using the Allred score, which is a semiquantitative system that takes into consideration the proportion of positive cells (scored on a scale of 0 to 5) and staining intensity (scored on a scale of 0 to 3). The proportion of positive cells and the staining intensity were then summed to produce total scores of 0 or 2 to 8. A score of 0 to 2 was regarded as negative, while 3 to 8 was seen as positive. Her 2 status was evaluated as negative $(0$ and $1+)$ or positive $(2+$ and $3+)$. E-cadherin immunoreactivity of the tumor and normal tissues was assessed by considering the predominant expression pattern, as follows: normal (complete membrane staining), aberrant (cytoplasmic and heterogeneous staining), or absent (no staining).

For fluorescence in situ hybridization (FISH), $2 \mu \mathrm{m}$-thick sections from each paraffin block were prepared. Deparaffinization, pretreatment, and protease digestion were performed as described by the FGFR2/CEN10p FISH probe kit protocol (Abnova Corp., Taipei, Taiwan). Probes were hybridized at $37^{\circ} \mathrm{C}$ for 14 to 18 hours. After hybridization, the slides were washed in $2 \times \mathrm{SSC} / 0.3 \% \mathrm{NP}-40$ at $72^{\circ} \mathrm{C}$ for 5 minutes, air-dried, and counterstained with 4,6-diamidino-2-phenylindole (DAPI). The slides were examined under a fluorescence microscope (Olympus BX51; Olympus, Tokyo, Japan) equipped with Spectrum Texas Red, fluorescein isothiocyanate, and DAPI filters. The slides were stored at $-20^{\circ} \mathrm{C}$ until examination. The FGFR2/CEN10p ratio was determined on the basis of counts of at least 40 cells by enumerating both red (FGFR2) and green (CEN10p on chromosome 10) signals. Samples with a FGFR2/CEN1Op ratio greater than 2 were considered to exhibit FGFR2 amplification. All histology, IHC staining, and FISH results were evaluated by two pathologists (M.J.S. and Y.S.P.).

\section{Assessment of Helicobacter pylori infection}

H. pylori infection status was investigated by IHC staining (lot \#215A-76; Cell Marque, Rocklin, CA, USA) and by reviewing the medical records. Normal tissues for IHC staining were available in 14 patients in the case group and 32 patients in the control group. In addition, one patient in the case group and one patient in the control group who had a medical history of $H$. pylori eradication before the diagnosis of stomach cancer were also considered to be $H$. pylori-positive regardless of the IHC staining results.

\section{Statistical analysis}

The case and control groups were compared in terms of categorical and continuous variables using Fisher exact test and Mann-Whitney U test, respectively. The variables were expressed as median with interquartile range (IQR) or patient numbers with percentage. Cumulative survival was determined using the Kaplan-Meier method. The difference between the two groups in terms of survival was assessed using the log-rank test. The correlations between clinicopathological variables and overall survival were assessed using Cox proportional hazard regression analysis. The $\mathrm{p}$-values less than 0.05 were considered to indicate statistical significance. All data analyses were performed using IBM SPSS version 18.0 (SPSS Inc., Chicago, IL, USA).

\section{RESULTS}

\section{Clinical features of gastric cancer patients who were diagnosed during pregnancy}

Of the 583 patients who were diagnosed with cancer during pregnancy, 20 had gastric cancer. These 20 patients formed the case cohort of the study. Their median age was 31 years (IQR, 29.0 to 33.8 years). Seventeen patients were diagnosed during pregnancy, and their median gestational age at diagnosis was 30 weeks (IQR, 15.8 to 37.3 weeks). The remaining three patients were diagnosed postpartum at 3, 10, and 28 days, respectively. The most common clinical presentation was abdominal pain (five cases, 25\%) followed by nausea and/or vomiting (four cases, 20\%), nonspecific dyspepsia (three cases, 15\%), and melena (one case, 5\%). Distant metastatic features, including abdominal distention due to ascites, a palpable mass, and jaundice, were identified in seven patients (35\%). Curative gastrectomy was the most common intervention (six cases, 30\%), followed by adjuvant chemotherapy (four patients, 20\%). Twelve patients (70\%) received palliative therapy. The median survival time of all 20 patients was 7.0 months (IQR, 2.0 to 23.8 months). However, the median survival time of those who underwent gastrectomy was 32.5 months (IQR, 21.8 to 76.0 months) compared with 6.2 months (IQR, 1.0 to 7.0 months) for those who received palliative chemotherapy and 2.1 months (IQR, 1.0 to 13.5 months) for those who were given best supportive care only. The median time from the onset of symptoms to diagnosis was 77.4 days (IQR, 30 to 142.5 days), and the time to treatment from pathological diagnosis was 21 days (IQR, 9.0 to 30.5 days). Seven patients terminated their pregnancy. The median gestational age at termination was 8 weeks (IQR, 7 to 23 weeks). The remaining 13 patients maintained their pregnancy and delivered live fetuses. The median gestational age at delivery was 35 weeks (IQR, 30.5 to 38.0 weeks). No obstetric complications were reported. Fetal metastasis was not identified. However, one of the 11 patients whose placental specimens were obtained showed 
Table 2. Clinicopathological Features of the Gastric Cancer in the Pregnancy-Associated and Control Groups

\begin{tabular}{|c|c|c|c|}
\hline Clinicopathological feature & Case $(n=20)$ & Control $(n=39)$ & p-value \\
\hline Age, yr & $31(29-33.8)$ & $31(29-34)$ & $0.927^{*}$ \\
\hline Family history of gastric cancer ${ }^{\dagger}$ & $1(4.8)$ & $2(4.9)$ & $1.000^{\ddagger}$ \\
\hline Helicobacter pylori infection ${ }^{\S}$ & $6 / 15(40)$ & $8 / 33(24.2)$ & $0.315^{\ddagger}$ \\
\hline Time from symptom to diagnosis, day" & $77.4(30-142.5)$ & $66(28-208)$ & $0.751^{*}$ \\
\hline Time from diagnosis to treatment, day" & $21(9-30.5)$ & $7(3.5-16)$ & $0.021^{*}$ \\
\hline Initial presentation* & & & $0.087^{\ddagger}$ \\
\hline Abdominal pain & $5(25)$ & $8(21.6)$ & \\
\hline Nausea/vomiting & $4(20)$ & $2(5.4)$ & \\
\hline Dyspepsia & $3(15)$ & $18(48.6)$ & \\
\hline Bleeding & $1(5)$ & $2(5.4)$ & \\
\hline Metastatic symptoms & $7(35)$ & $7(18.9)$ & \\
\hline Tumor location & & & $0.006^{\ddagger}$ \\
\hline Antrum & $7(35)$ & $3(7.7)$ & \\
\hline Corpus & $7(35)$ & $27(69.2)$ & \\
\hline Entire & $6(30)$ & 9 (23.1) & \\
\hline Gross tumor type & & & $0.758^{\ddagger}$ \\
\hline Early gastric cancer & $2(10)$ & $4(10.3)$ & \\
\hline Advanced gastric cancer & $18(90)$ & $35(89.7)$ & \\
\hline Ulcerofungating (Borrmann II) & $1(5.6)$ & $2(5.7)$ & \\
\hline Ulceroinfiltrative (Borrmann III) & 7 (38.9) & $19(54.3)$ & \\
\hline Diffuse infiltrative (Borrmann IV) & $10(55.6)$ & $14(40)$ & \\
\hline Clinical or pathological stage & & & $1.000^{\ddagger}$ \\
\hline I & $1(5)$ & $2(5.1)$ & \\
\hline II & $3(15)$ & $6(15.4)$ & \\
\hline III & $2(10)$ & $4(10.3)$ & \\
\hline IV & $14(70)$ & $27(69.2)$ & \\
\hline Treatment summary & & & $0.376^{\ddagger}$ \\
\hline Gastrectomy & $2(10)$ & $3(7.7)$ & \\
\hline Gastrectomy+adjuvant chemotherapy & $4(20)$ & $14(35.9)$ & \\
\hline Palliative therapy & $8(40)$ & $17(43.6)$ & \\
\hline Best supportive care & $6(30)$ & 5 (12.8) & \\
\hline Histological differentiation & & & $0.663^{\ddagger}$ \\
\hline Adenocarcinoma poorly differentiated & $10(50)$ & $23(59.0)$ & \\
\hline Signet ring cell carcinoma & $10(50)$ & $15(38.5)$ & \\
\hline Mucinous carcinoma & 0 & $1(2.6)$ & \\
\hline Lauren classification & & & $0.661^{\ddagger}$ \\
\hline Intestinal & 0 & $1(2.6)$ & \\
\hline Diffuse & $20(100)$ & $38(97.4)$ & \\
\hline \multicolumn{4}{|l|}{ IHC staining and FISH ${ }^{\pi}$} \\
\hline ER-positive & $1 / 17$ (5.9) & $0 / 31(0)$ & $0.446^{\ddagger}$ \\
\hline PR-positive & $1 / 17(5.9)$ & 0/31 (0) & $0.446^{\ddagger}$ \\
\hline EGFR-positive & $1 / 17(5.9)$ & $3 / 31(9.7)$ & $0.891^{\ddagger}$ \\
\hline Her2-positive & 0/17 (0) & $2 / 31(6.5)$ & $0.645^{\ddagger}$ \\
\hline Loss of E-cadherin & 9/17 (52.9) & $18 / 38(47.4)$ & $0.103^{\ddagger}$ \\
\hline FGFR2 amplification & $1 / 17(5.9)$ & 0/22 (0) & $0.436^{\ddagger}$ \\
\hline Overall survival, mo & 7 (2-23.8) & $15(6-41)$ & $0.189^{\#}$ \\
\hline
\end{tabular}

Data are presented as median (IQR) or number (\%).

IQR, interquartile range; IHC, immunohistochemistry; FISH, fluorescence in situ hybridization; ER, estrogen receptor; PR, progesterone receptor; EGFR, epidermal growth factor receptor; Her2, human epidermal growth factor receptor 2; FGFR2, fibroblast growth factor receptor 2.

${ }^{*}$ Mann-Whitney U test; ${ }^{\dagger}$ First-degree relatives; ${ }^{\ddagger}$ Fisher exact test; ${ }^{\circledR}$ Not available in all cases because of the lack of available normal gastric tissue or clinical records; "Not available in all cases because of the lack of clinical information; "Not available in all cases because of tumor tissue loss;

${ }^{\#}$ Log rank according to the Kaplan-Meier method. 
microscopic placental metastasis (case number 19, Table 1). The clinical features and obstetric and oncological outcomes of the case patients are summarized in Table 1.

\section{Clinicopathological features of the gastric cancers in the case and control groups}

To identify factors that affected the prognosis of pregnancyassociated gastric cancer, age-, sex-, and stage-matched patients with gastric cancer that did not associate with pregnancy were identified from the same medical database. The cases and controls are compared in terms of their characteristics in Table 2. The median overall survival of the cases and controls was 7.0 and 15.0 months, respectively ( $\mathrm{p}=0.189$ ) (Fig. $1 \mathrm{~A})$. Abdominal pain and dyspepsia were the most common initial presentation in the case and control groups, respectively $(p=0.087)$. In terms of the tumor location, the corpus was the most prevalent epicenter of the tumor in the control group (69.2\%), whereas the antrum, corpus, and entire stomach were equally often the epicenter of the tumor in the case group (35\%, 35\%, and 30\%, respectively, $\mathrm{p}=0.006$ ). In both groups, the gastric cancers were mostly advanced in terms of gross type (90\% and $89.7 \%$ in the case and control groups, respectively) and clinical stage (stage IV; $70 \%$ and $69.2 \%$ in the case and control groups, respectively). Curative surgery with or without adjuvant chemotherapy was performed in 30\% and 43.6\% of the case and control group patients, respectively. In both groups, almost all cancers were poorly differentiated or signet ring cell carcinomas. The case group was more likely to be infected with $H$. pylori $(6 / 15,40 \%)$ than the control group $(8 / 33,24.2 \%)$, but this difference did not achieve statistical significance $(\mathrm{p}=0.315)$. More than half $(57.1 \%)$ of all $H$. pylori-infected patients had cancer in the antrum. Although the two groups did not differ significantly in terms of time from the appearance of symptoms to diagnosis (77.4 and 66 days in the case and control groups, respectively; $p=0.242$ ),

\section{A}

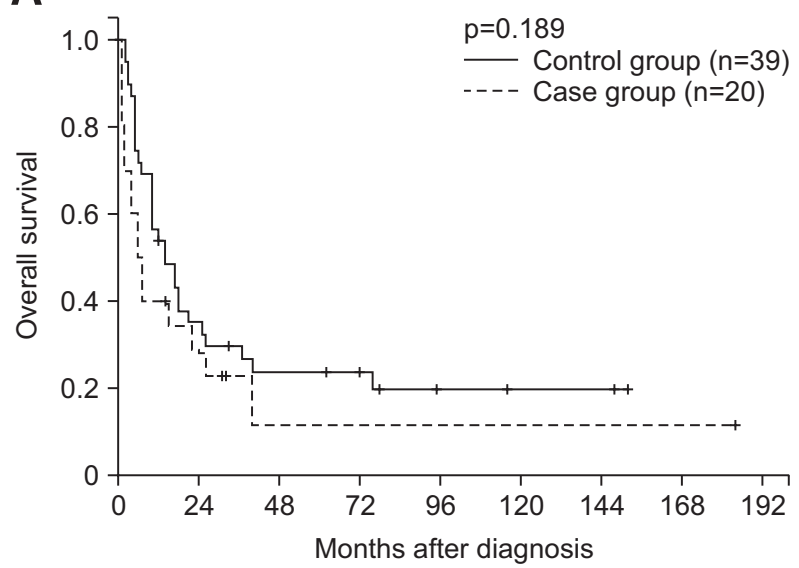

the case group had a significantly longer time from diagnosis to treatment ( 21 days vs 7 days, $p=0.021$ ). None of the patients had prior history of endoscopy before the diagnosis of the gastric cancer.

The case and control groups did not differ significantly in terms of IHC and FISH results. Only one case (5.9\%) and no controls were immunopositive for ER. This was also observed for PR immunopositivity and positivity for FGFR2 amplification. One case (5.9\%) and three controls (9.7\%) were immunopositive for EGFR. None of these four cases were immunopositive for more than one marker. None of the cases but two of the controls (6.5\%) were immunopositive for Her2. Loss of E-cadherin expression was identified in 52.9\% of the case group and $47.4 \%$ of the control group.

\section{Factors that associate significantly with survival}

The pregnancy-associated group and the whole (case plus control) cohort were then analyzed to identify factors that associated with survival. In the pregnancy-associated group, a tumor stage of IV (compared with stages I, II, and III) associated with the highest risk of death (adjusted hazard ratio [aHR], 86.701; 95\% confidence intervals [CI], 1.047 to 7,178.283; $\mathrm{p}=0.048$ ), followed by a postpartum diagnosis (compared with diagnosis in the first trimester: aHR, 23.802; 95\% CI, 2.499 to 226.727; $\mathrm{p}=0.006$ ) and tumor that involved the entire stomach (compared with tumor in the antrum: aHR, 4.834; 95\% CI, 1.256 to 18.604 ; $\mathrm{p}=0.022$ ). Diagnosis in the third trimester also tended to associate with a poorer prognosis compared with diagnosis in the first trimester (aHR, 5.440; 95\% CI, 1.003 to 29.512) with marginal statistical significance $(\mathrm{p}=0.05)$. Age, loss of E-cadherin, and other clinicopathological factors did not associate with overall survival in this analysis (Table 3).

When the whole study cohort (cases plus controls) were analyzed, tumor stage again had prognostic significance (aHR,

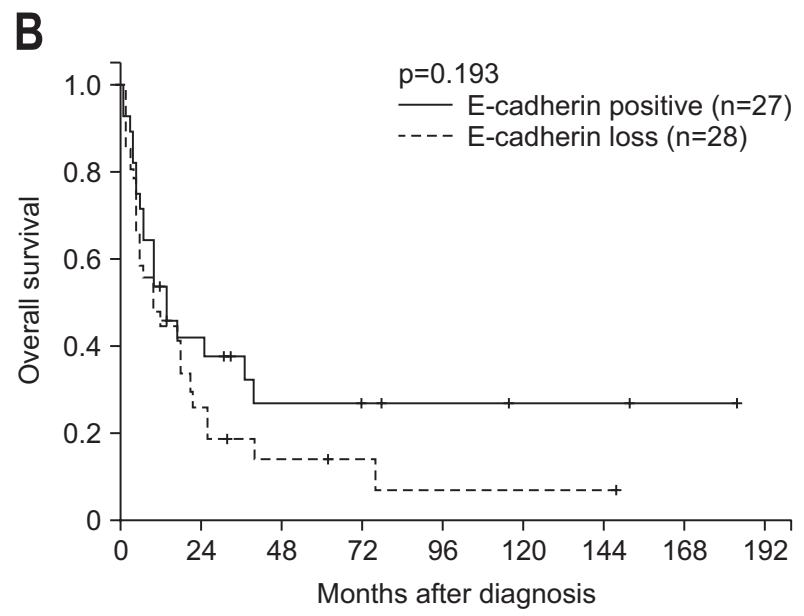

Fig. 1. Kaplan-Meier survival curves according to (A) the status of pregnancy and (B) E-cadherin expression. Not all cases were available for Ecadherin immunostaining because of tissue loss. 
Table 3. Identification of the Prognostic Factors for Overall Survival in Pregnancy-Associated Gastric Cancers Alone and in All Cases Evaluated in This Study

\begin{tabular}{|c|c|c|c|c|}
\hline \multirow{2}{*}{ Factor } & \multicolumn{2}{|c|}{ Pregnancy-associated GC } & \multicolumn{2}{|c|}{ All GC } \\
\hline & $\mathrm{HR}(95 \% \mathrm{CI})$ & $\mathrm{p}$-value & HR $(95 \%$ CI) & p-value \\
\hline Age & $0.939(0.843-1.046)$ & 0.939 & $0.985(0.923-1.051)$ & 0.655 \\
\hline Pregnancy & - & - & $1.490(0.809-2.745)$ & 0.200 \\
\hline \multicolumn{5}{|c|}{ Gestational period at diagnosis } \\
\hline 1st trimester & Reference & Reference & - & - \\
\hline 2nd trimester & $3.933(0.69-22.409)$ & 0.123 & - & - \\
\hline 3rd trimester & $5.440(1.003-29.512)$ & 0.050 & - & - \\
\hline Postpartum & 23.802 (2.499-226.727) & 0.006 & - & - \\
\hline Stage (I, II, III vs IV) & $86.701(1.047-7178.283)$ & 0.048 & $12.149(4.602-32.073)$ & $<0.001$ \\
\hline \multicolumn{5}{|l|}{ Tumor location } \\
\hline Antrum & Reference & Reference & Reference & Reference \\
\hline Corpus & $2.185(0.574-8.316)$ & 0.252 & $2.674(1.020-7.011)$ & 0.045 \\
\hline Entire & $4.834(1.256-18.604)$ & 0.022 & $3.344(1.918-9.336)$ & 0.021 \\
\hline Loss of E-cadherin & $1.380(0.449-4.238)$ & 0.574 & $1.498(0.819-2.742)$ & 0.190 \\
\hline
\end{tabular}

GC, gastric cancer; HR, hazard ratio; CI, confidence interval.

12.149; 95\% CI, 4.602 to 32.073 ; $p<0.001)$. A location in either the corpus or the entire stomach also associated significantly with a poor prognosis (corpus location: aHR, 2.674, 95\% CI, 1.020 to 7.011, p=0.045; entire stomach: aHR, 3.344, 95\% CI, 1.918 to $9.336, \mathrm{p}=0.021$ ). The loss of E-cadherin tended to associate with an unfavorable prognosis but failed to achieve statistical significance (Fig. 1B). Most notably, although the cases had an overall shorter survival than the controls, pregnancy did not associate significantly with prognosis (Fig. 1A).

\section{DISCUSSION}

In the present case-control study, 20 patients with pregnancyassociated gastric cancer were compared with 39 age-, sex-, and stage-matched control cases with gastric cancer. The prognosis of pregnancy-associated gastric cancer was grave: the median overall survival time was 7 months. Our findings showed that patients with pregnancy-associated gastric cancer had a worse prognosis if their tumor stage was advanced, the tumor was located in the entire stomach, or the diagnosis was made in the 2 month postpartum period. A diagnosis in the third trimester also tended to associate with a worse prognosis. All of the patients who were diagnosed during the third trimester or the postpartum period had stage IV disease (10/10). The median time from diagnosis to treatment was longer in the case group (21 days) than in the control group (7 days). This may reflect the fact that the symptoms of gastric cancer frequently overlap with pregnancy-associated symptoms such as hyperemesis and gastric discomfort, which are easily presumed to be secondary to normal pregnancy. More importantly, expecting mothers tend to hesitate when faced with the need for immediate treatment be- cause the surgery or chemotherapy could harm the fetus. Moreover, patients who are diagnosed late in the second trimester or early in the third trimester may delay treatment to rescue their child from the complications caused by preterm birth. However, comparison of the cases with the control patients, who lacked these complicating factors, showed that this delay in the treatment did not affect the outcome of the case patients (Table 2, Fig. 1A). We speculate that the longer duration from diagnosis to treatment of the cases did not significantly affect their outcome because they were already in advanced stage when they were diagnosed and, as shown in Table 3, tumor stage was by far the most important factor in terms of prognosis (Table 3).

A significant proportion of the patients in the case and control groups had cancers that involved the entire stomach $(30 \%$ and 21\%, respectively), indicating Borrmann type IV cancers. We also discovered that the cases were significantly more likely than the controls to have the tumor in the antrum (35\% vs 7.7\%). This was not entirely expected because we also found that the cases tended to have $H$. pylori infection more frequently than the controls, although this difference did not achieve statistical significance: antral tumors are more likely to associate with $H$. pylori infection, which is a major cause of gastric cancer in older patients. However, it should be noted that previous studies have suggested that while the gastric cancers in older patients generally associate with prolonged exposure to carcinogens such as $H$. pylori infection and the accumulation of numerous genetic changes, gastric cancer in younger patients (including pregnancy-associated cases) may be driven by a few dominant genetic carcinogenic alterations or a certain hormonal milieu. ${ }^{12}$ This conflicting result with literature was also observed in a recent Korean study, ${ }^{7}$ and it would be interesting to investigate 
the correlation of $H$. pylori infection and pregnancy.

Our survival analyses of the whole cohort (cases plus controls) showed that advanced stage and tumors located in the corpus or entire stomach were significant poor prognostic factors (Table 3). Similar to previous studies, ${ }^{5,7}$ we found that early detection and curative resection were the most important and effective approaches in pregnancy-associated gastric cancers. It should be noted that while it is generally believed that gastric cancers in younger patients are more aggressive than those in older patients, Santoro et al. ${ }^{13}$ have shown that the prognosis of younger patients with gastric cancer is not different from that of older patients with the same stage disease. In addition, two other studies have shown that younger patients with gastric cancer could actually have a better prognosis than older patients, especially in operable cases. ${ }^{3,9}$ Therefore, appropriate and careful measures should be taken to identify and treat gastric cancers, including when the patient is younger and pregnant.

Two studies have shown that gastric cancers in younger patients differ from conventional gastric cancers in terms of clinicopathological characteristics. ${ }^{14,15}$ Therefore, we subjected the tumor tissues of the cases and controls in this study to IHC or FISH to assess whether they exhibit overexpression or amplification of well-studied growth factor receptors, namely, ER, PR, EGFR, Her2, and FGFR2. It has been reported that ER immunopositivity associates with diffuse-type/poorly differentiated carcinomas or Borrmann type IV cancers; some studies suggest that estrogen has a trophic effect on gastric cancer cell lines, whereas, others assert that estrogen has a protective role. ${ }^{13,14,16-18}$ EGFR and Her2 overexpression associates with gastric cancer ${ }^{19,20}$ and thus these molecules are targets for pharmaceuticals whose efficacy in gastric cancer has been assessed by several clinical trials. ${ }^{21,22}$ FGFR2 is another therapeutic target in gastric carcinoma ${ }^{23}$ since it has been reported that FGFR2 amplification or overexpression occurs more frequently in diffuse-type gastric cancers that have a poor prognosis. ${ }^{24}$ However, in our study, the pregnancy-associated and control groups did not differ significantly in terms of the expression or amplification of these markers. This suggests that these growth factors may associate with age rather than with pregnancy. To confirm this hypothesis, these growth factors should be tested in older sex- and stage-matched patients with gastric cancer. Notably, we found one case of FGFR2 amplification in the pregnancy-associated group: the same patient also had placental metastasis.

We also assessed whether the cases and controls differed in terms of the E-cadherin loss because it has been shown that germline missense mutations in $\mathrm{CDH} 1$ in hereditary diffuse gastric cancers associate with functional inactivation of E-cadherin $^{25}$ (although it should be pointed out that fewer than 10\% of younger patients with gastric cancer have a family history of gastric cancer ${ }^{26}$ ). We found that E-cadherin expression was lost in $52.9 \%$ of the pregnancy-associated group and $47.4 \%$ of the control group. This is significantly higher than the frequencies of E-cadherin loss reported in older patients with gastric cancer. ${ }^{15}$ However, although we observed that the patients whose tumors expressed E-cadherin tended to have slightly better outcomes than those who had lost E-cadherin expression, this difference did not achieve statistical significance (Fig. 1B). A recent study reported that somatic mutation and deletions of Ecadherin, rather than E-cadherin expression, are prognostic factors in gastric cancer. The authors of that study also could not detect a strong correlation between genetic aberration of $\mathrm{CDH} 1$ and E-cadherin expression. ${ }^{27}$ Thus, it may be more important to analyze genetic aberrations of $\mathrm{CDH} 1$ than to evaluate Ecadherin expression.

Our study has some limitations. First, it has a retrospective observational design that is based on a cohort from a single center. Factors known to be important to survival, such as the details of adjuvant and/or palliative chemotherapy, were also underestimated in this study. A future comprehensive metaanalysis may produce more useful information in terms of the optimal management of pregnancy-associated gastric cancer. Although we analyzed only a handful of biomarkers, highthroughput analysis using next generation sequencing could greatly enhance the quality and quantity of information regarding the genetic alterations that associate with this disease.

In summary, the prognosis of pregnancy-associated gastric cancer associated with gestational age at diagnosis, advanced stage, and tumor location. Although the prognosis is dismal, our study indicates the importance of the early detection and curative resection of gastric cancer in these patients. Given that the pregnancy-associated cases did not differ significantly from the control patients in terms of the expression or amplification of growth factor receptors (ER, PR, EGFR, Her2, and FGFR2) and the expression of E-cadherin, these markers may not associate with pregnancy-related gastric cancer.

\section{CONFLICTS OF INTEREST}

No potential conflict of interest relevant to this article was reported.

\section{ACKNOWLEDGEMENTS}

\section{Author's contributions:}

Min Jeong Song, analysis and interpretation of data, drafting the manuscript. Se Jeong Park, analysis and interpretation of data. Young Soo Park and Ho June Song, study conception and design, analysis and interpretation of data, critical revision for important intellectual content, approval of the final version of the manuscript. Ji Yong Ahn, Kee Don Choi, Gin Hyug Lee, Hwoon-Yong Jung, Jeong Hwan Yook, and Byung Sik Kim, collection, assembly, analysis of data, approval of the final version of the manuscript, material and technical support. 


\section{REFERENCES}

1. Crew KD, Neugut AI. Epidemiology of gastric cancer. World J Gastroenterol 2006;12:354-362.

2. Jemal A, Siegel R, Ward E, et al. Cancer statistics, 2006. CA Cancer J Clin 2006;56:106-130.

3. Al-Refaie WB, Hu CY, Pisters PW, Chang GJ. Gastric adenocarcinoma in young patients: a population-based appraisal. Ann Surg Oncol 2011;18:2800-2807.

4. Sakamoto K, Kanda T, Ohashi M, et al. Management of patients with pregnancy-associated gastric cancer in Japan: a mini-review. Int J Clin Oncol 2009;14:392-396.

5. Ueo H, Matsuoka H, Tamura S, Sato K, Tsunematsu Y, Kato T. Prognosis in gastric cancer associated with pregnancy. World J Surg 1991;15:293-297.

6. Kodama I, Takeda J, Koufuji K, Yano S, Hanzawa M, Shirouzu K. Gastric cancer during pregnancy. Kurume Med J 1997;44:179183.

7. Lee HJ, Lee IK, Kim JW, Lee KU, Choe KJ, Yang HK. Clinical characteristics of gastric cancer associated with pregnancy. Dig Surg 2009;26:31-36.

8. Suh M, Choi KS, Lee YY, Park B, Jun JK. Cancer screening in Korea, 2012: results from the Korean National Cancer Screening Survey. Asian Pac J Cancer Prev 2013;14:6459-6463.

9. Lai JF, Kim S, Li C, et al. Clinicopathologic characteristics and prognosis for young gastric adenocarcinoma patients after curative resection. Ann Surg Oncol 2008;15:1464-1469.

10. Saito H, Takaya S, Fukumoto Y, Osaki T, Tatebe S, Ikeguchi M. Clinicopathologic characteristics and prognosis of gastric cancer in young patients. Yonago Acta Med 2012;55:57-61.

11. Isobe T, Hashimoto K, Kizaki J, et al. Characteristics and prognosis of gastric cancer in young patients. Oncol Rep 2013;30:43-49.

12. Tavares A, Gandra A, Viveiros F, Cidade C, Maciel J. Analysis of clinicopathologic characteristics and prognosis of gastric cancer in young and older patients. Pathol Oncol Res 2013;19:111-117.

13. Santoro R, Carboni F, Lepiane P, Ettorre GM, Santoro E. Clinicopathological features and prognosis of gastric cancer in young European adults. Br J Surg 2007;94:737-742.

14. Park HJ, Ahn JY, Jung HY, et al. Clinical characteristics and outcomes for gastric cancer patients aged 18-30 years. Gastric Cancer 2014;17:649-660.

15. Milne AN, Carvalho R, Morsink FM, et al. Early-onset gastric cancers have a different molecular expression profile than conven- tional gastric cancers. Mod Pathol 2006;19:564-572.

16. Harrison JD, Watson S, Morris DL. The effect of sex hormones and tamoxifen on the growth of human gastric and colorectal cancer cell lines. Cancer 1989;63:2148-2151.

17. Jaspers VK, Gillessen A, Quakernack K. Gastric cancer in pregnancy: do pregnancy, age or female sex alter the prognosis? Case reports and review. Eur J Obstet Gynecol Reprod Biol 1999;87:1322.

18. Palli D, Cipriani F, Decarli A, et al. Reproductive history and gastric cancer among post-menopausal women. Int J Cancer 1994;56: 812-815.

19. Oh HS, Eom DW, Kang GH, et al. Prognostic implications of EGFR and HER-2 alteration assessed by immunohistochemistry and silver in situ hybridization in gastric cancer patients following curative resection. Gastric Cancer 2014;17:402-411.

20. Moelans CB, Milne AN, Morsink FH, Offerhaus GJ, van Diest PJ. Low frequency of HER2 amplification and overexpression in early onset gastric cancer. Cell Oncol (Dordr) 2011;34:89-95.

21. Kim YH, Sasaki Y, Lee KH, et al. Randomized phase II study of nimotuzumab, an anti-EGFR antibody, plus irinotecan in patients with 5-fluorouracil-based regimen-refractory advanced or recurrent gastric cancer in Korea and Japan: preliminary results. J Clin Oncol 2011;29(4 Suppl):87.

22. Bang YJ, Van Cutsem E, Feyereislova A, et al. Trastuzumab in combination with chemotherapy versus chemotherapy alone for treatment of HER2-positive advanced gastric or gastro-oesophageal junction cancer (ToGA): a phase 3, open-label, randomized controlled trial. Lancet 2010;376:687-697.

23. Matsumoto K, Arao T, Hamaguchi T, et al. FGFR2 gene amplification and clinicopathological features in gastric cancer. Br J Cancer 2012;106:727-732.

24. Toyokawa T, Yashiro M, Hirakawa K. Co-expression of keratinocyte growth factor and K-sam is an independent prognostic factor in gastric carcinoma. Oncol Rep 2009;21:875-880.

25. Suriano G, Oliveira C, Ferreira P, et al. Identification of CDH1 germline missense mutations associated with functional inactivation of the E-cadherin protein in young gastric cancer probands. Hum Mol Genet 2003;12:575-582.

26. Kokkola A, Sipponen P. Gastric carcinoma in young adults. Hepatogastroenterology 2001;48:1552-1555.

27. Corso G, Carvalho J, Marrelli D, et al. Somatic mutations and deletions of the E-cadherin gene predict poor survival of patients with gastric cancer. J Clin Oncol 2013;31:868-875. 